\title{
Eficiência dos municípios de Mato Grosso do Sul: uma abordagem baseada em fronteira determinística
}

\author{
Efficiency of the municipalities of Mato Grosso do Sul State: an approach based \\ in deterministic frontier
}

\author{
Eficiencia de los municipios de Mato Grosso do Sul: un enfoque basado en \\ frontera determinística
}

\author{
Antônio Carlos Cantero Dorsa ${ }^{1}$ \\ José Carlos Taveira ${ }^{1}$ \\ Mariana Souza Pereira ${ }^{1}$ \\ Frederico Kochhann Santos ${ }^{2}$ \\ Reginaldo Brito Costa ${ }^{1}$
}

\begin{abstract}
Recebido em 29/10/2019; revisado e aprovado em 03/12/2019; aceito em 18/02/2019
DOI: http://dx.doi.org/10.20435/inter.v21i3.2788
\end{abstract}

\begin{abstract}
Resumo: O presente artigo objetivou investigar a eficiência nos gastos públicos dos municípios do estado de Mato Grosso do Sul - Brasil, compreendendo o período entre 2005 e 2016. Para a realização das análises, foram consideradas as variáveis: gastos com administração, saúde, educação, Produto Interno Bruto per capita (PIBpc), a população municipal e o Índice Firjan de Desenvolvimento Municipal. O método de cálculo da eficiência utilizado foi a Análise Envoltória de Dados (DEA). Os resultados empíricos mostraram eficiências variadas entre os municípios, dentro do período proposto, e foram apontadas no mapa as eficiências médias municipais, que mostraram sete municípios na faixa I; 15 municípios na faixa II; 17 municípios na faixa III; e 16 municípios na faixa IV. A partir desses resultados, não podemos afirmar que um nível maior de eficiência pode levar a um aumento ou diminuição do Índice Firjan de Desenvolvimento, concluindo que há, em média, ineficiência na gestão dos recursos públicos, e esses não garantem resultados na melhoria da renda, longevidade e educação dos sul-mato-grossenses.
\end{abstract}

Palavras-chave: DEA; eficiência; recursos públicos; desenvolvimento.

Abstract: This article aimed to investigate the efficiency in public spending of the municipalities of the state of Mato Grosso do Sul - Brazil, covering the period between 2005 and 2016. For the analysis, we considered the following variables: administration expenses, health, education, Gross Domestic Product per capita (GDPpc), the municipal population, and the Firjan Municipal Development Index. We used as an efficiency calculation method the Data Envelopment Analysis (DEA). The empirical results showed varied efficiencies among the municipalities, within the proposed period, and the average municipal efficiencies on the map were pointed out, which showed seven municipalities in the range I; 15 municipalities in the range II; 17 municipalities in range III; and 16 municipalities in range IV. From these results, we cannot say that a higher level of efficiency can lead to an increase or decrease in the Firjan Development Index, concluding that there is inefficiency in the management of public resources, and these do not guarantee results in improving income, longevity, and education of Mato Grosso do Sul.

Keywords: DEA; efficiency; public resources; development.

Resumen: Este artículo tuvo como objetivo investigar la eficiencia en el gasto público de los municipios del estado de Mato Grosso do Sul - Brasil, cubriendo el período comprendido entre 2005 y 2016 . Para el análisis, se consideraron las siguientes variables: gastos de administración, salud, educación, Producto Interno Bruto per cápita (PIBPc), la población municipal y el Índice de Desarrollo Municipal de Firjan. El método de cálculo de eficiencia utilizado fue el Análisis de Envoltura de Datos (DEA). Los resultados empíricos mostraron eficiencias variadas entre los municipios, dentro del período propuesto, y fueran señaladas en el mapa las eficiencias municipales promedio, que mostraron siete municipios en el rango I; 15 municipios en el rango II; 17 municipios en el rango III; y 16 municipios en el rango IV. A partir de estos resultados, no se puede decir que un mayor nivel de eficiencia puede conducir a un aumento o disminución del Índice de Desarrollo de Firjan, concluyendo que hay ineficiencia en la gestión de los recursos públicos, y esos no garantizan resultados en la mejora de los ingresos, la longevidad y la educación de Mato Grosso do Sul. Palabras clave: DEA; eficiencia; recursos públicos; desarrollo.

\footnotetext{
${ }^{1}$ Universidade Católica Dom Bosco (UCDB), Campo Grande, Mato Grosso do Sul, Brasil.

${ }^{2}$ Faculdade Estácio de Sá, Campo Grande, Mato Grosso do Sul, Brasil.
} 


\section{INTRODUÇÃO}

A eficiência na gestão pública pode garantir qualidade de vida para a população e é um dos principais objetivos do desenvolvimento do milênio, bem como uma das principais metas que as nações devem priorizar, a fim de garantir o acesso aos direitos fundamentais para toda a sociedade.

Os municípios são as estruturas públicas mais próximas das pessoas, executando ações e gerenciando recursos públicos disponíveis pelos três níveis governamentais. Os entes públicos são regidos pela Lei de Responsabilidade Fiscal (LRF), em vigor desde 2000, que obriga uma maior transparência na prestação de contas públicas e exige uma melhor qualidade na gestão, como preceitua a própria Carta Magna, dando uma ênfase destacada na eficiência como um princípio constitucional.

Nesse contexto, investigar a eficiência relativa da gestão dos recursos no nível municipal é um dos propósitos para comparar as melhores formas de uso e aplicação da arrecadação municipal. Um dos indicadores de análise é o Índice Firjan ${ }^{3}$ de Desenvolvimento Municipal (IFDM), que acompanha anualmente o desenvolvimento socioeconômico de todos os mais de cinco mil municípios brasileiros em três áreas de atuação: Emprego e Renda; Educação; e Saúde. Porém o Índice Firjan não analisa a eficiência relativa, que é o propósito deste estudo, de conhecer quais municípios de Mato Grosso do Sul (MS) usa melhor seus recursos em relação aos demais.

O campo empírico de estudo está localizado na região Centro-Oeste do Brasil, que se tornou, desde o início do século, a maior região brasileira produtora de soja, milho e carne bovina e o maior centro de exportação, com um papel importante no fornecimento de alimentos para o mundo. Mato Grosso do Sul é um dos maiores produtores de grãos e cana-de-açúcar, além de alta produtividade e competitividade da pecuária; porém está entre os estados com mais elevada relação receita-despesa e um dos maiores gastos com pessoal do Brasil.

A partir desse cenário, o presente artigo objetivou investigar a eficiência nos gastos públicos dos municípios do estado de Mato Grosso do Sul, no período entre 2005 e 2016.

A metodologia usada para o cálculo da eficiência foi a Análise Envoltória de Dados (DEA) e se deve a Farrell (1957), em seu artigo seminal. O modelo DEA constrói uma fronteira determinística, que posiciona as unidades produtivas consideradas eficientes em uma dada amostra.

Os pioneiros no uso do DEA foram Charnes, Cooper e Rhodes (1978), cujos fundamentos são discutidos no trabalho seminal de Farrell (1957). Uma fronteira de eficiência não paramétrica da amostra de dados é criada usando técnicas de programação linear. Portanto a fronteira é construída pelas práticas (combinação de entradas e saídas) das unidades produtivas mais eficientes da amostra. O problema de programação linear consiste na maximização da relação ponderada entre a saída e a entrada da empresa.

Os modelos DEA clássicos são o CCR (CHARNES; COOPER; RHODES, 1978) e o BCC (BANKER; CHARNES; COOPER, 1984). O modelo CCR, apresentado originalmente por Charnes, Cooper e Rhodes (1978), constrói uma superfície linear por partes não paramétrica, envolvendo os dados e trabalhando com retornos constantes de escala, isto é, qualquer variação nas entradas (inputs) produz variação proporcional nas saídas (outputs).

Para o alcance do objetivo, além desta introdução e das conclusões, o artigo está dividido nos seguintes tópicos: 1) materiais e método, com dados e estatísticas descritivas das variáveis e da eficiência; 2) resultados relativos às regiões de Mato Grosso do Sul e 3) as discussões.

\footnotetext{
${ }^{3}$ Federação das Indústrias do Estado do Rio de Janeiro.
} 


\section{MATERIAIS E MÉTODOS}

O método DEA permite, a partir da programação matemática, elaborar uma fronteira determinística de eficiência relativa, usando dados de entrada (inputs) e saídas (output). Ele avalia os municípios que utilizaram os recursos de forma mais eficiente ou menos eficiente, apresentando um índice de 0 a 1, sendo 1 para a maior eficiência.

O método DEA estabelece comparações entre o que foi produzido (denominado output), considerando-se os recursos (ou insumos) de cada município (denominados inputs). Trata-se de uma metodologia de análise de eficiência que compara o resultado otimizado com a eficiência de cada unidade de gestão municipal.

Para o presente artigo, a abordagem não paramétrica, utilizada no método DEA, foi definida por um output (Índice Firjan) e cinco inputs (gastos com administração, com saúde, com educação, o PIBpc e a população do município). O modelo de análise utilizado foi o DEA-BCC, apresentado por Banker, Charnes e Cooper (1984), que considera retornos variáveis de escala, isto é, substitui o axioma da proporcionalidade entre inputs e outputs pelo axioma da convexidade. Por isso, esse modelo também é conhecido como Variable Returns to Scale (VRS).

\subsection{Dados}

A presente pesquisa tem caráter exploratório e abordagem de análise quantitativa. O estudo utiliza séries temporais entre 2005 e 2016, com dados por municípios de MS de bases oficiais, como o Sistema IBGE de Recuperação Automática (Sidra), da Secretaria do Tesouro Nacional, e o Índice Firjan de Desenvolvimento Municipal.

Uma das variáveis utilizadas na pesquisa foi o PIBpc, obtido no Sidra. A Secretaria do Tesouro Nacional foi a base de dados das despesas por município nas áreas de Administração, Saúde, Educação, a partir de sua população total por ano.

O IFDM é um estudo do Sistema Firjan, que acompanha anualmente o desenvolvimento socioeconômico de todos os mais de cinco mil municípios brasileiros em três áreas de atuação: Emprego e Renda; Educação; e Saúde. Ele foi criado em 2008 e é feito, exclusivamente, com base em estatísticas públicas oficiais, disponibilizadas pelos ministérios do Trabalho, Educação e Saúde.

Do total dos 79 municípios de MS, o presente estudo excluiu 24 deles, por ausência de dados em algum ano ou em alguma das variáveis escolhidas para a análise. Os municípios excluídos foram: Água Clara, Aquidauana, Aral Moreira, Antônio João, Bandeirante, Bodoquena, Bonito, Bela Vista, Costa Rica, Caarapó, Douradina, Dois Irmãos do Buriti, Eldorado, Guia Lopes da Laguna, Iguatemi, Ivinhema, Jateí, Ladário, Paraíso das Águas, Porto Murtinho, Sete Quedas, Tacuru, Três Lagoas e Vicentina.

Os 55 municípios analisados no estudo foram divididos de acordo com as nove regiões do estado de Mato Grosso do Sul, a saber:

» Região Norte - Alcinópolis, Camapuã, Coxim, Figueirão, Pedro Gomes, Rio Negro, Rio Verde de Mato Grosso, São Gabriel do Oeste, Sonora;

» Região Leste - Anaurilândia, Angélica, Bataguassu, Batayporã, Nova Andradina, Novo Horizonte do Sul, Taquarussu;

»Região do Pantanal - Anastácio, Corumbá, Miranda;

»Região Sudoeste - Caracol, Jardim, Nioaque;

» Região Sul-Fronteira - Amambai, Coronel Sapucaia, Laguna Carapã, Paranhos, Ponta Porã; 
» Região do Bolsão - Aparecida do Taboado, Brasilândia, Cassilândia, Chapadão do Sul, Inocência, Paranaíba, Santa Rita do Pardo, Selvíria;

»Região de Campo Grande - Campo Grande, Corguinho, Jaraguari, Nova Alvorada do Sul, Ribas do Rio Pardo, Rochedo, Sidrolândia, Terenos;

»Região do Cone Sul - Itaquiraí, Japorã, Juti, Mundo Novo, Naviraí;

»Região da Grande Dourados - Deodápolis, Dourados, Fátima do Sul, Glória de Dourados, Itaporã, Maracaju, Rio Brilhante.

\subsection{Estatística descritiva das variáveis utilizadas}

Na Tabela 1, são apresentadas as estatísticas descritivas das variáveis utilizadas na análise, que compreende os anos de 2005 a 2016, para 55 municípios de MS, apresentando a média anual e o desvio padrão de cada ano.

Tabela 1 - Estatísticas descritivas das variáveis utilizadas no modelo DEA VRS

\begin{tabular}{|c|c|c|c|c|c|c|c|}
\hline Ano & MUNIC. & INDFJ & POP. & ADM. & SAÚDE & EDUC. & PIBpc \\
\hline \multirow{2}{*}{2005} & Média & 0,63 & 34.179 & 4.960 .788 & 9.005 .462 & 8.429 .407 & 356.196 \\
\hline & Desvio padrão & 0,08 & 102.110 & 9.909 .879 & 32.881 .091 & 22.471 .707 & 1.011 .637 \\
\hline \multirow{2}{*}{2006} & Média & 0,64 & 34.735 & 5.630 .594 & 10.910 .246 & 9.951 .288 & 399.948 \\
\hline & Desvio padrão & 0,08 & 104.207 & 11.265 .419 & 40.256 .343 & 26.976 .728 & 1.164 .073 \\
\hline \multirow{2}{*}{2007} & Média & 0,65 & 34.094 & 7.330 .897 & 13.337 .953 & 12.560 .033 & 453.257 \\
\hline & Desvio padrão & 0,09 & 98.821 & 14.520 .224 & 49.604 .193 & 32.764 .382 & 1.313 .369 \\
\hline \multirow{2}{*}{2008} & Média & 0,65 & 35.157 & 7.595 .939 & 16.592 .761 & 15.263 .262 & 548.195 \\
\hline & Desvio padrão & 0,09 & 101.874 & 14.760 .312 & 61.299 .523 & 41.195 .663 & 1.539 .070 \\
\hline \multirow{2}{*}{2009} & Média & 0,67 & 35.544 & 7.885 .625 & 17.792.073 & 16.040 .151 & 591.250 \\
\hline & Desvio padrão & 0,09 & 102.960 & 15.990 .782 & 64.132 .963 & 44.659 .024 & 1.742 .548 \\
\hline \multirow{2}{*}{2010} & Média & 0,68 & 36.818 & 8.421 .643 & 21.156 .618 & 18.066 .277 & 700.113 \\
\hline & Desvio padrão & 0,08 & 107.234 & 17.295 .724 & 73.369 .679 & 48.721 .141 & 2.062 .911 \\
\hline \multirow{2}{*}{2011} & Média & 0,68 & 37.259 & 10.521 .333 & 23.634 .493 & 20.629 .185 & 818.684 \\
\hline & Desvio padrão & 0,08 & 108.526 & 23.865 .724 & 85.464 .119 & 56.726 .582 & 2.417 .983 \\
\hline \multirow{2}{*}{2012} & Média & 0,69 & 37.684 & 11.211 .823 & 27.393 .621 & 24.557 .445 & 916.722 \\
\hline & Desvio padrão & 0,08 & 109.776 & 25.235 .905 & 100.235 .872 & 66.100 .242 & 2.640 .259 \\
\hline \multirow{2}{*}{2013} & Média & 0,70 & 38.924 & 10.677 .171 & 28.546 .784 & 24.878 .915 & 998.443 \\
\hline & Desvio padrão & 0,07 & 113.460 & 19.377 .615 & 104.385 .642 & 65.873 .579 & 2.847 .807 \\
\hline \multirow{2}{*}{2014} & Média & 0,70 & 39.424 & 12.076 .468 & 32.630 .881 & 28.258 .585 & 1.146 .417 \\
\hline & Desvio padrão & 0,08 & 114.934 & 22.508 .362 & 118.332 .829 & 77.510 .317 & 3.288 .627 \\
\hline \multirow{2}{*}{2015} & Média & 0,69 & 39.424 & 10.999 .080 & 35.254 .312 & 31.377 .750 & 1.186 .614 \\
\hline & Desvio padrão & 0,07 & 114.934 & 13.146 .472 & 132.379 .719 & 91.518 .122 & 3.349 .136 \\
\hline \multirow{2}{*}{2016} & Média & 0,70 & 39.912 & 12.167 .623 & 37.555 .095 & 34.276 .327 & 1.263 .217 \\
\hline & Desvio padrão & 0,06 & 116.371 & 13.681 .090 & 137.441 .218 & 97.419 .795 & 3.519 .074 \\
\hline
\end{tabular}

Fonte: Elaboração própria, a partir de dados secundários do Sidra-IBGE (2018) e do Índice Firjan (2019).

Verificou-se um alto desvio padrão nas variáveis população, PIBpc, gastos com educação, saúde e administração, que pode ser explicado pela diferença populacional e locacional entre a capital e os pequenos municípios. As grandes distâncias entre os municípios são uma característica do estado de Mato Grosso do Sul, por possuir 70\% do Pantanal e ter a maior parte das fazendas de monocultura com extensões médias maiores do Brasil. 
O Índice Firjan médio da série de anos estudados foi de 0,67, sendo classificado como um índice moderado de desenvolvimento. Além de ter Índice Firjan médio em relação aos demais estados, os dados das receitas de Mato Grosso do Sul têm alta variação pelo porte dos municípios e pelas diferentes atividades econômicas e sua matriz de insumo-produto.

\subsection{Estatística descritiva da eficiência por ano}

Na Tabela 2, a seguir, são apresentadas as médias, o desvio padrão, o valor mínimo, o valor máximo e a mediana dos 55 municípios estudados, no período de 2005 a 2016.

Tabela 2 - Estatística Descritiva da Eficiência por ano

\begin{tabular}{|c|c|c|c|c|c|c|}
\hline Ano & Obs. & Média & Dv. Pd. & Mín. & Máx. & Mediana \\
\hline 2005 & 55 & 0,54 & 0,30 & 0,04 & 1 & 0,48 \\
\hline 2006 & 55 & 0,49 & 0,29 & 0,04 & 1 & 0,44 \\
\hline 2007 & 55 & 0,46 & 0,28 & 0,04 & 1 & 0,42 \\
\hline 2008 & 55 & 0,38 & 0,21 & 0,04 & 0,88 & 0,37 \\
\hline 2009 & 55 & 0,38 & 0,20 & 0,04 & 1 & 0,34 \\
\hline 2010 & 55 & 0,36 & 0,20 & 0,04 & 0,9 & 0,32 \\
\hline 2011 & 55 & 0,33 & 0,21 & 0,04 & 1 & 0,30 \\
\hline 2012 & 55 & 0,35 & 0,25 & 0,03 & 1 & 0,30 \\
\hline 2013 & 55 & 0,32 & 0,21 & 0,03 & 1 & 0,28 \\
\hline 2014 & 55 & 0,29 & 0,20 & 0,03 & 1 & 0,26 \\
\hline 2015 & 55 & 0,25 & 0,17 & 0,03 & 0,77 & 0,23 \\
\hline 2016 & 55 & 0,29 & 0,24 & 0,03 & 1 & 0,22 \\
\hline
\end{tabular}

Fonte: Elaboração própria, a partir de dados secundários do Sidra-IBGE (2018) e do Índice Firjan (2019).

Os resultados apontam que os municípios operaram com baixa eficiência ao longo do período analisado, e a média caiu de 0,54 em 2005 para 0,29 em 2016, indicando, assim, uma diminuição da eficiência nos gastos públicos municipais.

\section{RESULTADOS}

O modelo DEA-BCC utilizado maximiza o Índice Firjan em relação às demais variáveis, e seus resultados em média foram apresentados na Figura 1, que mostra o mapa das eficiências médias dos municípios do estado de Mato Grosso do Sul, no qual se constata que nenhum município foi considerado totalmente eficiente no presente estudo, ou seja, nenhum obteve índice de 1,0. Os municípios foram divididos dentro de sua faixa de eficiência, ou seja, Faixa I de 0 a 0,25, Faixa II de 0,26 a 0,50, Faixa III de 0,51 a 0,75 e Faixa IV de 0,75 a 1. Salienta-se, ainda, que os municípios da cor branca que apresentam índice de 0 como resultado são os excluídos do estudo por ausência de dados. 
Figura 1 - Mapa das eficiências médias dos municípios

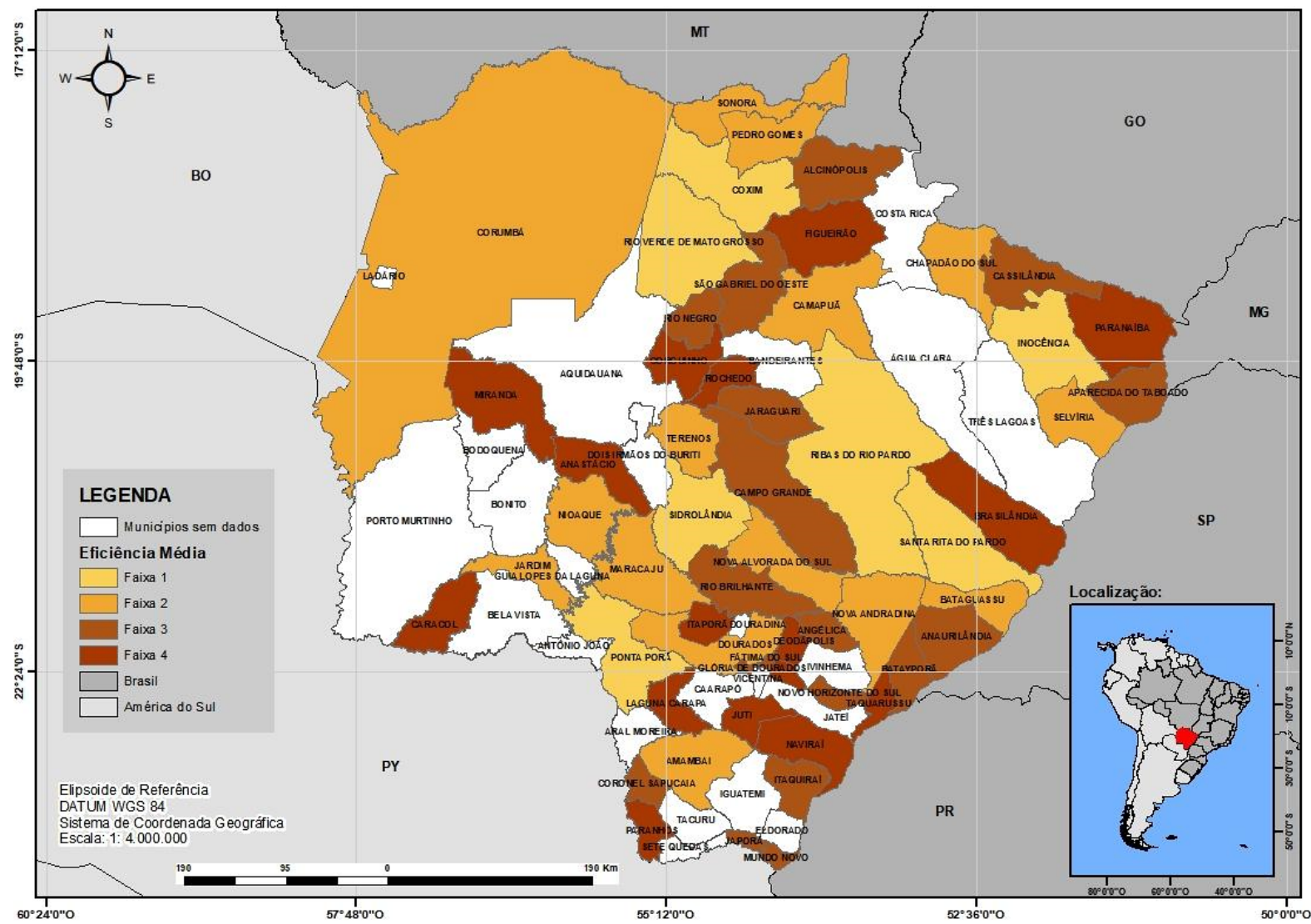

Fonte: Elaboração própria, a partir de dados secundários do Sidra (IBGE, 2018).

De maneira a contribuir de forma espacial, foi dividida a amostra em regiões de acordo com os critérios da Secretaria de Desenvolvimento Estadual de MS, e a eficiência foi calculada e analisada para as nove regiões do estado de MS, mostrando a eficiência média de cada município dentro da sua respectiva região do estado. A primeira região é uma das maiores, pois, além de incluir a capital de MS, une oito municípios distintos e que apresentam, juntos, mais de $40 \%$ da população do estado de MS.

\subsection{Região de Campo Grande}

Na Figura 2, são apresentadas as eficiências dos municípios da região de Campo Grande no período analisado no estudo. 
Figura 2 - Região de Campo Grande

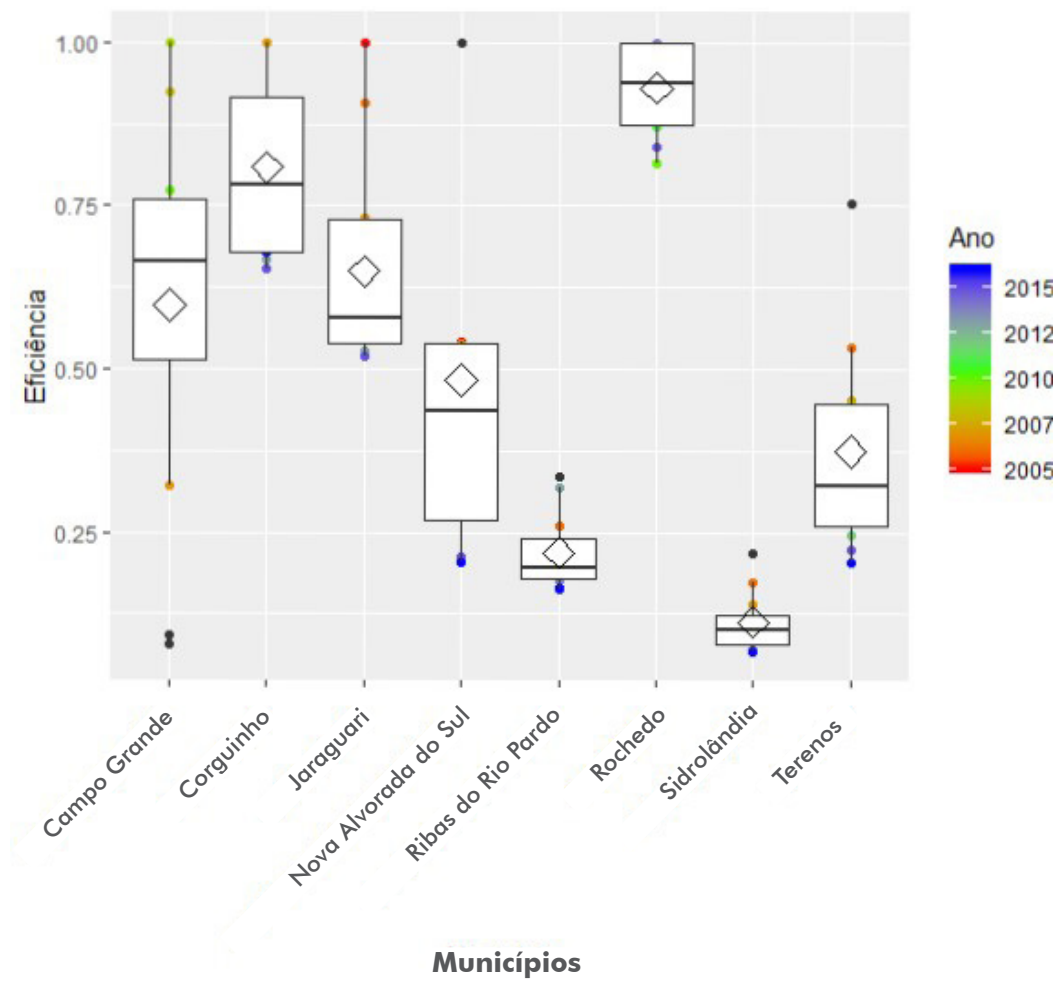

Fonte: Elaboração própria, a partir de dados secundários do Sidra (IBGE, 2018).

Os municípios da Região de Campo Grande apresentaram, em sua maioria, índices de eficiência variados. Rochedo, com média de 0,93, e Corguinho, com média de 0,80, apresentaram os mais altos índices da região.

O pequeno município de Jaraguari, com 0,65, e a capital do estado, Campo Grande, com 0,60, mostraram índices médios; em contraponto, Nova Alvorada do Sul $(0,48)$ e Terenos $(0,37)$ foram eficiências baixas. Por fim, Ribas do Rio Pardo $(0,22)$ e Sidrolândia $(0,11)$ apresentaram índices baixíssimos de eficiência média no período analisado. Esses municípios têm $43 \%$ da população do estado de MS, aproximadamente um milhão de habitantes, e a capital, Campo Grande, 35\% do total do PIBpc.

A eficiência média da região no período foi de 0,52, corroborando com os estudos de Oliveira e Liboni (2019), que encontraram resultados correlatos em seu estudo na mesorregião de Campinas, SP.

\subsection{Região do Bolsão}

Na Figura 3, são apresentadas as eficiências dos municípios da região do Bolsão no período analisado no estudo. 
Figura 3 - Região do Bolsão



Fonte: Elaboração própria, a partir de dados secundários do Sidra (IBGE, 2018).

A região do Bolsão apontou médias distintas de eficiência em seus municípios, com índices altos em Paranaíba $(0,90)$ e Brasilândia $(0,80)$; médios, em Cassilândia $(0,65)$ e Aparecida do Taboado $(0,60)$; baixos, em Chapadão do Sul $(0,48)$ e Selvíria $(0,37)$; e baixíssimos, em Inocência $(0,22)$ e Santa Rita do Pardo $(0,11)$. Marinho (2003) apontou resultados semelhantes, com grande dispersão de resultados entre os municípios do estado do Rio de Janeiro. A eficiência média da região no período foi de 0,52, sua população no período de 2016 apresentava 143.806 habitantes, 5\% da população estadual, e seu PIBpc representava $7 \%$ do total estadual.

\subsection{Região do Cone Sul}

Na Figura 4, são apresentadas as eficiências dos municípios da região do Cone Sul no período analisado no estudo.

Figura 4 - Região do Cone Sul

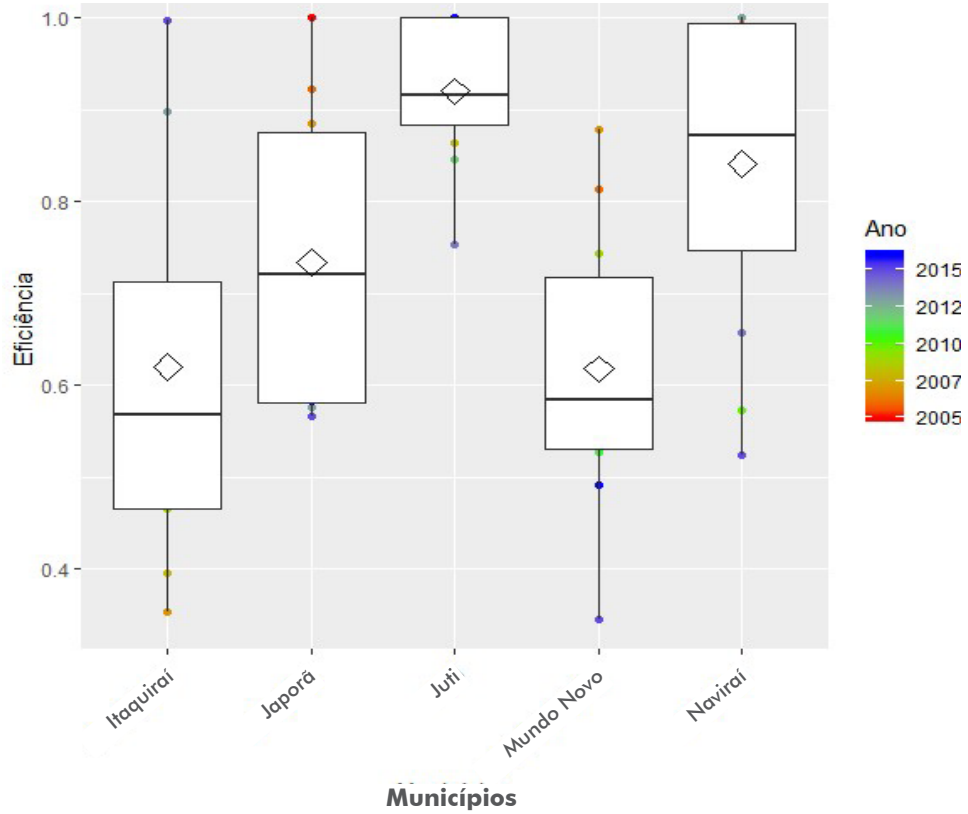

Fonte: Elaboração própria, a partir de dados secundários do Sidra (IBGE, 2018). 
De acordo com a Figura 4, os municípios de Juti, com média de eficiência alta, de 0,92, Naviraí $(0,84)$ e Japorã $(0,73)$ são os mais destacados na região do Cone Sul. Itaquiraí, com 0,62, e Mundo Novo, com 0,61, completam a região, mostrando índices médios de eficiência.

A população da região em 2016 era de 104.500 habitantes, 4\% do estado de MS, e seu PIBpc equivalia a 4\% do estadual. Pinto et. al (2015), em diagnóstico de preservação ambiental, apontam níveis bem parecidos de eficiência para essa região, assinalando como resultados índices médios.

\subsection{Região da Grande Dourados}

Na Figura 5, são apresentadas as eficiências dos municípios da região da Grande Dourados, no período analisado no estudo.

Figura 5 - Região da Grande Dourados

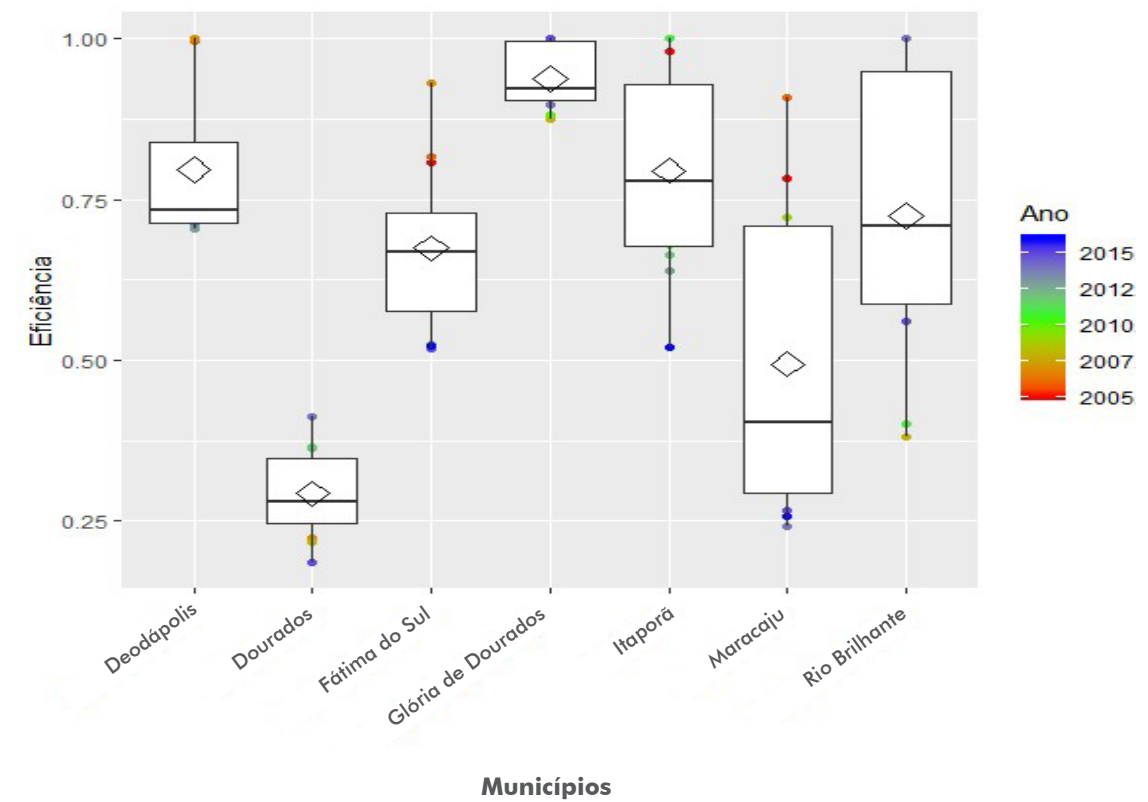

Fonte: Elaboração própria, a partir de dados secundários do Sidra (IBGE, 2018).

Os índices de eficiência médios dos municípios foram de Glória de Dourados $(0,94)$, seguido de Deodápolis $(0,80)$ e Itaporã $(0,79)$, como os mais altos. Na sequência, Rio Brilhante, com 0,72, e Fátima do Sul, com 0,67. Os mais baixos da região foram Maracaju $(0,50)$ e Dourados $(0,30)$.

O índice médio de eficiência na região da Grande Dourados, no período analisado, foi de 0,67. A população da região em 2016 era de 355.482 habitantes, o que equivalia a 14\% do total do estado, e seu PIBpc correspondia a 19\%. Souza et al. (2017) usaram o mesmo método nessa região, mas mediram a eficiência técnica de produtores de soja e analisaram os motivos que alguns produtores não se tornavam eficientes.

\subsection{Região Leste}

Na Figura 6, são apresentadas as eficiências dos municípios da região Leste, no período analisado no estudo. 


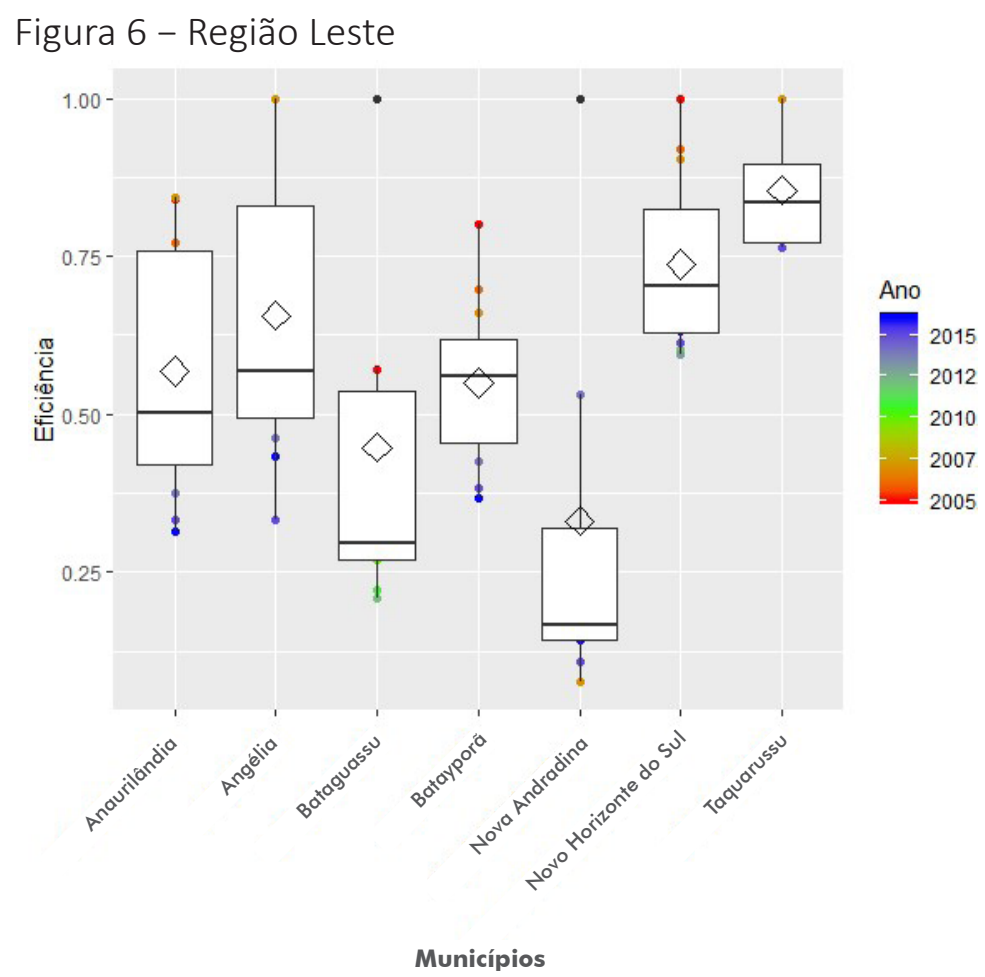

Fonte: Elaboração própria, a partir de dados secundários do Sidra (IBGE, 2018).

Os índices médios em cada município foram os seguintes: Taquarussu, com 0,85, e Novo Horizonte do Sul, com 0,74, indicando índice alto de eficiência; em seguida, Angélica $(0,66)$, Anaurilândia $(0,57)$, Batayporã $(0,55)$, Bataguassu $(0,45)$ e, com índice baixíssimo, Nova Andradina $(0,33)$.

A região Leste do estado de Mato Grosso do Sul apresentou um índice de eficiência médio no período analisado de 0,59, sendo sua população em 2016 de 110.745 habitantes, o que equivalia a $4 \%$ do total do estado, e seu PIBpc correspondia a $5 \%$ do total geral do estado.

Costa et al. (2013) mediram a eficiência dos recursos públicos à disposição das mesorregiões formadas pelos municípios do estado de Mato Grosso do Sul e apontaram que essas regiões têm capacidade de saldar suas dívidas.

\subsection{Região Norte}

Na Figura 7, são apresentadas as eficiências dos municípios da região Norte, no período analisado no estudo. 
Figura 7 - Região Norte

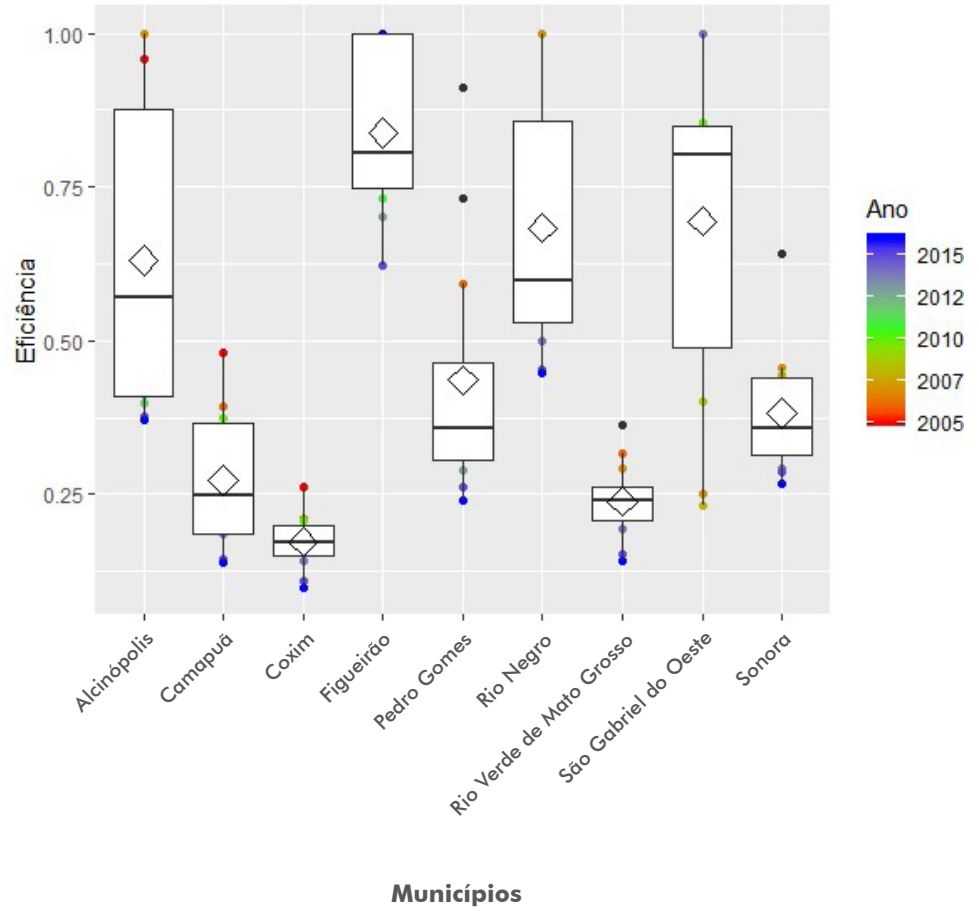

Fonte: Elaboração própria, a partir de dados secundários do Sidra (IBGE, 2018).

A média das eficiências foram: Figueirão $(0,84)$, São Gabriel do Oeste $(0,69)$, Rio Negro $(0,68)$, Alcinópolis $(0,63)$, Pedro Gomes $(0,43)$, Sonora $(0,38)$, Camapuã $(0,27)$, Rio Verde de Mato Grosso $(0,24)$ e Coxim $(0,17)$.

A região Norte do estado de Mato Grosso do Sul apresentou um índice de eficiência médio no período analisado de 0,48, sua população em 2016 era de 129.551 habitantes, correspondendo a 5\% do estado, e seu PIBpc equivalia, nesse período, a 6\% do total do estado. Richetti (2000) investigou os produtores de soja nessa região de Mato Grosso do Sul e apontou ótimos resultados de eficiência dos produtores, mesmo não operando com todo o potencial tecnológico disponível.

\subsection{Região do Pantanal}

Na Figura 8, são apresentadas as eficiências dos municípios da região do Pantanal, no período analisado no estudo. 


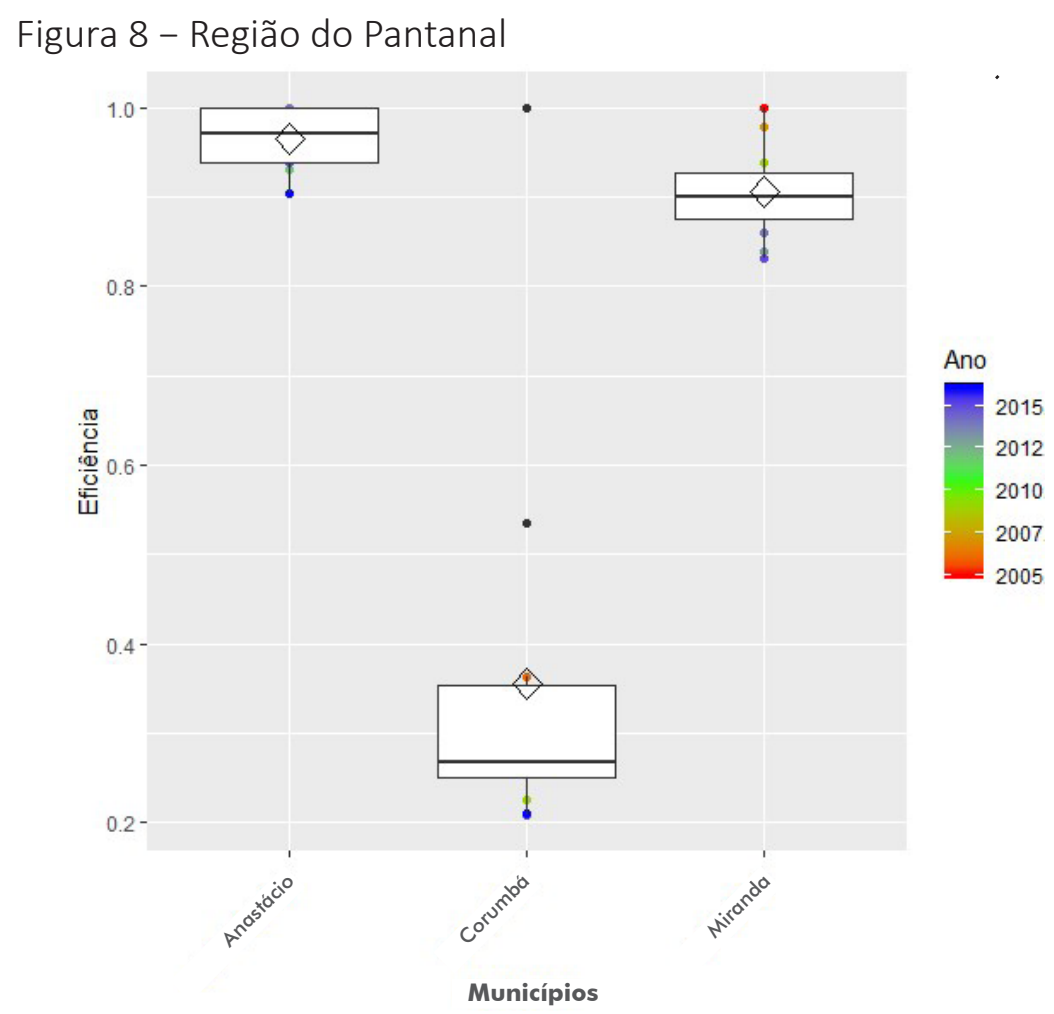

Fonte: Elaboração própria, a partir de dados secundários do Sidra (IBGE, 2018).

A média geral apresentou Anastácio (0,96), Miranda $(0,90)$ e Corumbá $(0,35)$, e a região do Pantanal apresentou um índice de eficiência médio de 0,74, sua população em 2016 era de 160.508 habitantes, representando $6 \%$ do estado e Corumbá, a maior cidade da região, com 108.000 habitantes, e seu PIBpc equivalia, nesse período, a 5\% do total do estado. Artigos correlatos, como o de Pinto et al. (2017), mediram a eficiência econômica dos assentamentos rurais do Pantanal Sul, que apresentaram bons índices de sustentabilidade, acompanhando os resultados de Anastácio e de Miranda.

\subsection{Região Sudoeste}

Na Figura 9, são apresentadas as eficiências dos municípios da região Sudoeste, no período analisado no estudo. 




Fonte: Elaboração própria, a partir de dados secundários do Sidra (IBGE, 2018).

Na eficiência média, Caracol é altíssima, com 0,95; e Jardim e Nioaque, baixos, com 0,48. A região Sudoeste apresentou um índice de eficiência médio de 0,63.

A população da região em 2016 era de 45.500 habitantes, representando 1,8\% do estado, e seu PIBpc equivalia, nesse período, a 1,2\% do total do estado.

Kaveski et al. (2015) concluíram que é considerada satisfatória a eficiência da utilização dos recursos destinados às unidades de saúde vinculadas ao SUS que são pertencentes ao Sudoeste de Mato Grosso do Sul.

\subsection{Região Sul-Fronteira}

Na Figura 10, são apresentadas as eficiências dos municípios da região Sul-Fronteira, no período analisado no estudo. 
Figura 10 - Região Sul-Fronteira

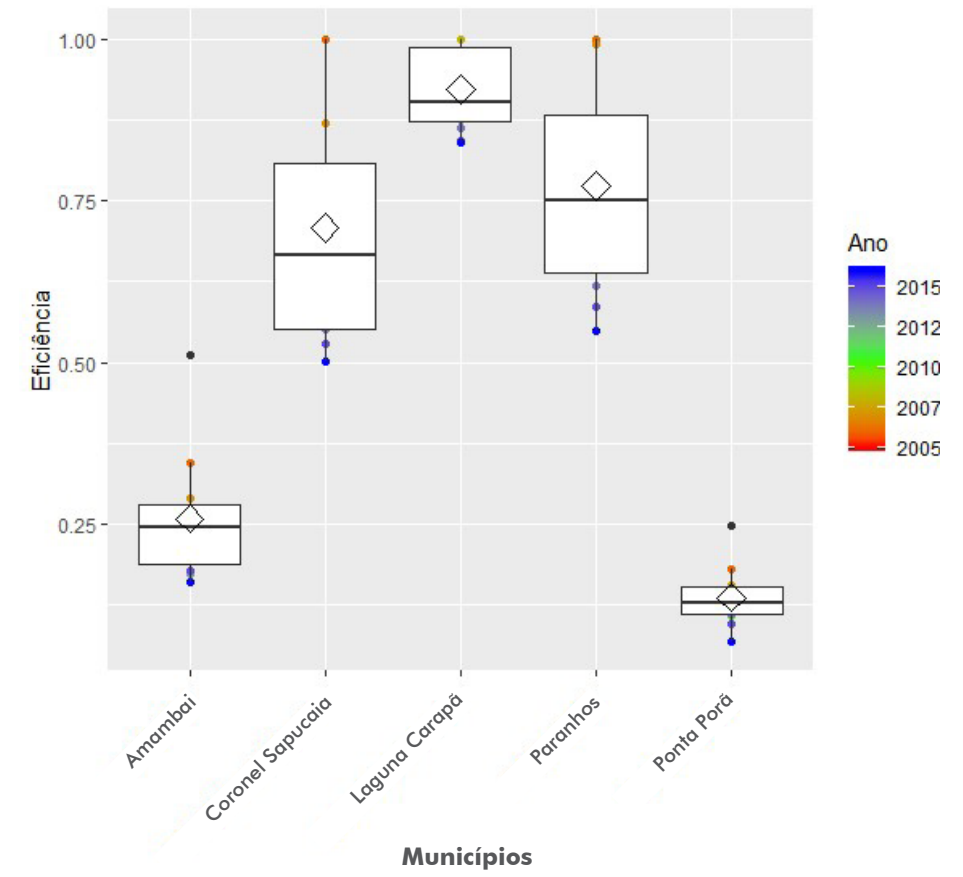

Fonte: Elaboração própria, a partir de dados secundários do Sidra (IBGE, 2018).

As médias de eficiência apontadas nesse período foram: Laguna Caarapã, com 0,92; Paranhos, 0,77; Coronel Sapucaia, 0,71; Amambai, 0,26; e Ponta Porã, 0,14. A média geral da região no período analisado foi de 0,56, e sua população em 2016 era de 160.000 habitantes, sendo Ponta Porã a cidade mais populosa, com 87.000 habitantes. A região representa $6 \%$ da população do estado, e seu PIBpc equivalia, nesse período, a 6\% do total do estado. Baptista et al. (2016) mostraram em seu estudo que a cidade de Ponta Porã tem alto grau de eficiência no setor turístico, corroborando a implementação de novas políticas públicas em favor desse nicho de mercado.

\section{DISCUSSÃO}

O comparativo geral entre as regiões é o seguinte: Cone Sul e Pantanal, com 0,75 e 0,74 , respectivamente, apontaram as melhores médias; em seguida, Grande Dourados $(0,67)$, Sudoeste $(0,63)$, Leste $(0,59)$, Sul-Fronteira $(0,56)$, Bolsão e Campo Grande $(0,52)$; e a região de menor eficiência é a Norte, com 0,48, o que pode indicar uma melhor gestão dos recursos públicos nas regiões com melhor desempenho medido. Os resultados apresentaram, ainda, após a divisão dos municípios em quatro faixas de eficiência: na faixa I, sete municípios ou 12,73\%; na faixa II, 15 municípios, ou seja, 27,27\%; na faixa III, 17 municípios, ou seja, 30,91\%; e, na faixa IV, 16 municípios obtiveram as maiores eficiências médias, ou seja, 29,09\% do total. Em relação à média do Índice Firjan, as faixas I e IV mostraram média de 0,65; a faixa III, de 0,67; e a faixa II, de 0,70, sendo a mais alta. Os resultados apontam que não podemos afirmar correlação entre as médias de eficiência e do Índice Firjan, ou seja, uma maior ou menor eficiência não tem relação com o aumento ou a diminuição do Índice Firjan.

Artigos correlatos merecem destaque, como os de Silva e Crisóstomo (2019), Catelli e Santos (2004), Prata e Arruda (2007), Silva Filho et al. (2016), Begnini e Tosta (2017), Ferri et 
al. (2017), Frio et al. (2018), Guedes (2002), Mesquita Monte e Bezerra Leopoldino (2017), Pereira et al. (2018), Sousa e Stošic (2005), os quais utilizaram a análise envoltória de dados para avaliar a gestão pública no Brasil.

Em Mato Grosso do Sul, Lima et al. (2018) e Lima et al. (2016) analisaram as eficiências dos assentamentos e de pequenas propriedades rurais, respectivamente. Castelão, Souza e Frainer (2016) identificaram as zonas ecológicas econômicas que se destacam quanto ao desenvolvimento sustentável e quais precisam melhorar, na ótica da eficiência. $\mathrm{Na}$ área da saúde, a análise envoltória de dados é bastante utilizada, seja na medida de internações realizadas no hospital, seja na capacidade ambulatorial do hospital, na quantidade de leitos e na sua média de permanência do paciente, ou também na produção ambulatorial e na capacidade dos equipamentos hospitalares, como visto nos trabalhos de Da Silva (2017), Vasconcelos et al. (2017) e Medeiros e Amaral Marcolino (2018).

Para medir eficiência das unidades produtivas, o estudo realizado por Torres et al. (2017) é indicado, pois avaliou a produção de peixes de pequenas pisciculturas e determinou a eficiência dos piscicultores, assim como o de Pinto et al. (2015), no qual diagnosticaram e avaliaram a eficiência da preservação do meio ambiente a partir da inclusão do pagamento de serviços ambientais, no estado de Mato Grosso do Sul.

O que foi observado nos resultados da metodologia DEA é a vasta utilização deste método nos mais variados segmentos e áreas, seja na Saúde, seja na Educação, Gestão Pública, Empreendimentos ou Meio Ambiente, o que comprova a importância e relevância da referida metodologia.

\section{CONCLUSÕES}

Os resultados medidos por região mostraram valores das médias do período (2005-2016) com diferentes níveis de eficiência. Destacaram-se os municípios de Anastácio $(0,96)$, Caracol $(0,95)$, Glória de Dourados $(0,94)$, Rochedo $(0,93)$, Laguna Caarapã e Juti $(0,92)$, Miranda e Paranaíba $(0,90)$, que apresentaram eficiências muito altas em relação aos demais.

Quanto às cidades mais populosas, Campo Grande, capital do estado, mostrou índice mediano de 0,60, já Dourados e Corumbá tiveram índices considerados baixos, de 0,30 e 0,35, respectivamente.

Os resultados sugerem a necessidade de melhor planejamento, e os gastos precisam de melhores ações e planejamento estratégico, pois, mesmo com o Índice Firjan médio do estado de MS acima do valor nacional, uma gestão de política pública mais efetiva pode levar a um maior desenvolvimento regional e local.

O presente artigo mostra relevante contribuição para a análise da gestão e eficiência dos entes públicos, uma vez que medir desempenho ainda é um tabu para as empresas públicas brasileiras, principalmente para as unidades municipais, que estão próximas das pessoas e devem atender às necessidades sociais, econômicas e ambientais da sociedade.

Para pesquisas futuras, o presente estudo deixa a lacuna de conhecer se choques aleatórios afetam a eficiência. Para esse fim, uma análise baseada em fronteira estocástica paramétrica pode ser uma ótima forma de atender essa pesquisa futura. 


\section{REFERÊNCIAS}

BAPTISTA, L. T. D.; PEREIRA, C. H.; SONAGLIO, C. M. Dinâmica do setor turístico em Ponta Porã-MS, Brasil. RITUR-Revista Iberoamericana de Turismo, v. 6, n. 2, p. 118-37, 2016.

BANKER, R.; CHARNES, A.; COOPER, W. W. Some models for estimating technical and scale inefficiencies in data envelopment analysis. Management Science, [S. I.], v. 30, n. 9, p. 1078-92, set. 1984.

BEGNINI, S.; TOSTA, H. T. A eficiência dos gastos públicos com a educação fundamental no Brasil: uma aplicação da análise envoltória de dados (DEA). Revista Economia \& Gestão, v. 17, n. 46, p. 43-59, 2017.

CASTELÃO, R. A.; SOUZA, C. C.; FRAINER, D. M. Nível de desenvolvimento sustentável dos municípios de Mato Grosso do Sul. Revista GeoPantanal, Corumbá, MS, v. 11, n. 20, p. 93-104, jan./jun. 2016.

CATELLI, A.; SANTOS, E. S. Mensurando a criação de valor na gestão pública. Revista de Administração Pública - RAP, Rio de Janeiro, v. 38, n. 3, p. 423-49, maio/jun. 2004.

CHARNES, A.; COOPER, W. W.; RHODES, E. Measuring the efficiency of decision making units. European journal of operational research, [S. I.], v. 2, n. 6, p. 429-44, nov. 1978.

COSTA, L. A.; HUPPES, C. M.; SOPRANE, G. A.; MACHADO, E. A. Comportamento da receita pública: uma análise dos recursos a disposição das mesorregiões formadas pelos municípios do Estado de Mato Grosso do Sul no ano de 2009. In: CONGRESSO BRASILEIRO DE CUSTOS, 20., 2013, Uberlândia. Anais [...]. Disponível em: https://anaiscbc.emnuvens.com.br/anais/article/viewFile/77/77. Acesso em: 24 jun. 2020.

DA SILVA, M. L.; PINTO, N. G. M.; CORNONEL, D. A.; SILVA, R. A. Análise da eficiência dos gastos públicos no Sistema Único de Saúde (SUS). Revista UNIFAMMA, v. 15, n. 2, 2016.

SILVA FILHO, G. M. DA; PEREIRA, T. R. L.; DANTAS, M. G. DA S.; ARAÚJO, A. O. Análise da eficiência nos gastos públicos com educação fundamental nos colégios militares do exército em 2014. Revista Evidenciação Contábil \& Finanças, v. 4, n. 1, p. 50-64, 2016.

FARRELL, M. The measurement of productive efficiency. Journal of the Royal Statistical Society. Series A (General), [S. I.], v. 120, n. 3, p. 253-90, 1957.

FEDERAÇÃO DAS INDÚSTRIAS DO ESTADO DO RIO DE JANEIRO (FIRJAN). Índice Firjan de Desenvolvimento Municipal (IFDM): consulta ao índice. Rio de Janeiro: Firjan, 2018. Disponível em: https://www.firjan.com. br/ifdm/. Acesso em: 10 set. 2019.

FERRI, C. M.; PEREIRA, R. S.; VIEIRA, S. F. A.; VISENTIN, I. C. A eficiência na alocação dos recursos de escolas municipais de Luziânia/GO: um estudo a partir da Análise Envoltória de Dados (DEA). In: ANAIS DO CONGRESSO BRASILEIRO DE CUSTOS-ABC, 14., Florianópolis, SC, 15 a 17 de novembro de 2017. Curitiba: Associação Brasileira de Custos, 2017.

FRIO, G. S.; FOCHEZATTO, A.; TRIACA, L. M.; FINN, E. S.; BRAATZ, J. Eficiência na educação: uma análise por escola no Rio Grande do Sul utilizando o método DEA em dois estágios. Revista Brasileira de Estudos Regionais e Urbanos, Curitiba, v. 12, n. 1, jul. 2018.

GUEDES, L. Uma análise da eficiência na formação de alunos dos cursos de engenharia civil das instituições de ensino superior brasileiras. 2002. Dissertação (Mestrado em Engenharia de Produção) - Universidade Federal do Rio de Janeiro, Rio de Janeiro, 2002. 
KAVESKI, I. D. S.; DEGENHART, L.; VOGT, M.; HEIN, N. A eficiência do atendimento nas Unidades de Saúde vinculadas ao SUS no sudoeste de Mato Grosso do Sul. Administração Pública e Gestão Social, Viçosa, MG, v. 7, n. 4, p. 197-205, out./dez. 2015.

LIMA, V. A. M. O.; SOUZA, C. C.; REIS NETO, J. F.; FRAINER, D. M. Análises das eficiências ambiental, econômica e social de assentamentos rurais em Mato Grosso do Sul (MS), através de análise envoltória de dados (DEA). Revista Ibero-Americana de Ciências Ambientais, [S. I.], v. 9, n. 4, p. 41-44, abr./maio 2018.

LIMA, V. A. M. O.; SOUZA, C. C.; REIS NETO, J. F.; LINS, R. S.; FRAINER, D. M. Análise da eficiência de pequenas propriedades rurais através do método da Análise Envoltória de Dados (DEA). Informe Gepec, Toledo, PR, v. 20, n. 2, p. 58-70, jul./dez. 2016.

MARINHO, A. Avaliação da eficiência técnica nos serviços de saúde nos municípios do Estado do Rio de Janeiro. Revista Brasileira de Economia, Rio de Janeiro, v. 57, n. 3, p. 515-34, jul./set. 2003.

MEDEIROS, R. V. V.; MARCOLINO, V. A. A Eficiência dos munícipios do Rio de Janeiro no setor de saúde: uma análise através da DEA e Regressão Logística. Revista Meta: Avaliação, v. 10, n. 28, p. 183-210, 2018.

MONTE, M. M.; LEOPOLDINO, C. B. Eficiência dos gastos municipais em educação no Ceará. Revista Pensamento Contemporâneo em Administração, v. 11, n. 4, p. 44-55, 2017.

OLIVEIRA, B. G.; LIBONI, L. B. Proposta de indicador de eficiência da gestão pública municipal em promover desenvolvimento local. Interações, Campo Grande, MS, v. 20, n. 3, p. 815-838, jul./set. 2019.

PEREIRA, R. S.; VIEIRA, S. F. A.; MADKUR, F. N. A Eficiência na alocação dos recursos de escolas municipais de São José dos Pinhais/PR: um estudo a partir da análise envoltória de dados (DEA). Revista Interdisciplinar de Gestão Social, v. 7, n. 3, 2018.

PINTO, J. S.; COSTA, E. A.; FRAINER, D. M.; OLIVEIRA, A. K. M.; SOUZA, C. C. Eficiência econômica dos assentamentos rurais do pantanal sul. Raega - O Espaço Geográfico em Análise, Curitiba, v. 40, p. 8-22, ago. 2017.

PINTO, J. S.; FRAINER, D. M.; OLIVEIRA, A. K. M.; SOUZA, C. C. Diagnóstico e avaliação da eficiência da preservação do ambiente em Mato Grosso do Sul a partir da inclusão do pagamento de serviços ambientais. Desenvolvimento e Meio Ambiente, Curitiba, v. 35, p. 225-40, dez. 2015.

PRATA, B. A.; ARRUDA, J. B. F. Aplicação da análise envoltória de dados na avaliação de eficiência de municípios: o caso do Estado do Ceará. In: SIMPÓSIO BRASILEIRO DE PESQUISA OPERACIONAL, 39., 2007, Fortaleza. Anais [...]. Fortaleza: SBPO, 2007. Disponível em: http://www.repositorio.ufc.br/bitstream/ riufc/12753/1/2007_eve_baprata_aplicacao.pdf. Acesso em: 24 jun. 2020.

RICHETTI, A. Fronteira de produção e eficiência econômica na cultura da soja no Mato Grosso do Sul. 2000. Dissertação (Mestrado em Administração Rural) - Universidade Federal de Lavras, Lavras, MG, 2000.

IBGE. Sistema IBGE de Recuperação Automática-Sidra. Rio de Janeiro: IBGE, 2018 Disponível em: https:// sidra.ibge.gov.br/Tabela/6579. Acesso em: 10 set. 2019.

SILVA, C. R. M.; CRISÓSTOMO, V. L. Gestão fiscal, eficiência da gestão pública e desenvolvimento socioeconômico dos municípios cearenses. Revista de Administração Pública, Rio de Janeiro, v. 53, n. 4, p. 791-801, jul./ago. 2019.

SOUSA, M. C. S.; STOŠIC, B. Technical e efficiency of the Brazilian municipalities: correcting nonparametric frontier measurements for outliers. Journal of Productivity Analysis, v. 24, n. 2, p. 157-81, 2005. 
SOUZA, C.; SILVA, F. A.; DEBOLETO, G. A. G.; CARVALHO, L. M.; REIS NETO, J. F.; FRAINER, D. M.; LEMES, L. H. B. Eficiência técnica de pequenos produtores de soja da região de Dourados (MS). Revista Espacios, Caracas, Venezuela, v. 38, n. 26, jan. 2017.

TORRES, S.; PEREIRA, F. A. R.; SOUZA, C. C.; FERREIRA, M. B. Análise da eficiência da produção da piscicultura na região de Dourados-MS. Revista Espacios, Caracas, Venezuela, v. 38, n. 52, 2017.

VASCONCELOS, M. I. O.; FARIAS, Q. L. T.; NASCIMENTO, F. G.; CAVALCANTE, A. S. P.; MIRA, Q. L. M.; QUEIROZ, M. V. O.. Educação em saúde na atenção básica: uma análise das ações com hipertensos. Revista de APS, v. 20, n. 2, 2017.

\section{Sobre os autores:}

Antônio Carlos Cantero Dorsa: Mestre em Desenvolvimento Local, Pós-Graduado Lato Sensu MBA Executivo em Gestão Empresarial e graduado em Administração Pública, todos pela Universidade Católica Dom Bosco (UCDB). Participante do Grupo de Pesquisa Science with R (SWR). Monitor Extensionista Peiex - 2019. E-mail: accdorsa@gmail.com, Orcid: http://orcid.org/0000-0002-2087-8772

José Carlos Taveira: Doutor em Desenvolvimento Local pela Universidad Complutense de Madrid, Espanha, diploma revalidado pela Universidade Federal do Rio Grande do Sul (UFRGS). Mestre em Matemática - Estatística pela Universidade Estadual Paulista "Júlio de Mesquita Filho" (Unesp). Especialista em Matemática pela Faculdade Auxilium de Lins, SP. Graduação em Engenharia Civil pela Universidade Federal de Mato Grosso do Sul (UFMS). Graduado em Matemática pela Universidade Católica Dom Bosco (UCDB). Graduação em Pedagogia pelas Faculdades Unidas Católica de Mato Grosso (FUCMT). Professor na Graduação e professor colaborador do Programa de Pós-Graduação (Mestrado e Doutorado) em Desenvolvimento Local (PPGDL) da UCDB. E-mail: proftave@gmail.com, Orcid: http://orcid.org/0000-0002-0967-2909

Mariana Souza Pereira: Mestranda do Programa de Pós-Graduação em Ciências Ambientais e Sustentabilidade Agropecuária (PPGCASA) da Universidade Católica Dom Bosco (UCDB). Graduada em Engenharia Sanitária e Ambiental pela UCDB. E-mail: marianapereira.esa@gmail.com, Orcid: http://orcid.org/0000-0002-9415-5374

Frederico Kochhann Santos: Mestre em Desenvolvimento Local pela Universidade Católica Dom Bosco (UCDB). Graduado em Publicidade e Propaganda pela Universidade AnhangueraUniderp. Docente, coordenador de curso de graduação e coordenador de pós-graduação da Faculdade Estácio de Sá (MS). Tem experiência na área de Comunicação Social, com ênfase em Publicidade e Propaganda, Jornalismo e Administração. E-mail: frederico.peiex@ucdb.br, Orcid: https://orcid.org/0000-0002-0144-117X

Reginaldo Brito Costa: Doutor em Ciências Florestais pela Universidade Federal do Paraná (UFPR). Mestre em Ciências Florestais pela Escola Superior de Agricultura Luiz de Queiroz (Esalq-USP). Graduado em Engenharia Florestal pela Universidade Federal de Mato Grosso (UFMT). Professor titular da Universidade Católica Dom Bosco. Tem experiência na área de Genética, com ênfase em Genética Quantitativa, atuando principalmente nos seguintes temas: desenvolvimento local, melhoramento genético, ganho genético, parâmetros genéticos. E-mail: reg.brito.costa@gmail.com, Orcid: http://orcid.org/0000-0002-5677-393X

Nota: Reginaldo Brito Costa e José Carlos Taveira participaram como avaliadores na banca de defesa da dissertação e orientação na metodologia e análise de resultados do artigo, e Mariana Pereira e Frederico Santos contribuíram no estudo do método Análise Envoltória de Dados (DEA), na coleta, limpeza dos dados, confecção dos gráficos e uso do software Rstudio. 